\title{
Diode-pumped femtosecond laser oscillator with cavity dumping
}

\author{
A. Killi and U. Morgner \\ Max-Planck-Institut für Kernphysik, \\ Saupfercheckweg 1, D-69117 Heidelberg, Germany, Tel: +49-6221-516338 \\ Fax: +49-6221-516604, email: Uwe.Morgner@mpi-hd.mpg.de \\ M. J. Lederer, D. Kopf \\ HighQLaser Production GmbH, \\ Kaiser-Franz-Josef-Str. 61, A-6845 Hohenems, Austria, \\ Tel.: + 43-5576 43040, Fax: + 43-5576-43050
}

\begin{abstract}
We report on a diode pumped tunable Yb:glass femtosecond laser oscillator with electro-optical cavity dumping. Pulses with energies exceeding $400 \mathrm{~nJ}$ and peak powers of above 1MW were generated at repetition frequencies as high as $200 \mathrm{kHz}$. This laser forms a compact light source for various scientific and industrial applications like micromachining.

(C) 2003 Optical Society of America

OCIS codes: (320.7090) Ultrafast lasers, (140.3580) Lasers, solid-state
\end{abstract}

Femtosecond laser systems have proven their potential in research laboratories in a variety of applications that were previously unthinkable, in fields as diverse as material processing, photonic device production, microscopy and biomedicine. The application of these lasers outside research laboratory environments has been, up to now, very limited because of their complexity and difficulty of operation and because of the high prices of commercially available laser systems. Cavity dumping is a possible way to generate pulses with energies sufficient for many applications like nonlinear frequency conversion [1], ultra-fast spectroscopy, and micro-machining [2,3] without using complex and expensive amplifier schemes. Stable pulses with various pulse lengths and energies have been obtained from cavity-dumped $\mathrm{TEM}_{00^{-}}$ pumped Ti:sapphire laser oscillators [4-7]. However, since Ti:sapphire based laser systems are not directly diode pumped and need complicated and expensive pump lasers, it is highly desirable to employ materials which are pumped directly by relatively inexpensive laser diodes. A very successful approach has been the use of Ytterbium doped materials in combination with semiconductor saturable absorber mirrors (SESAM) [8] to get high reliability and easy handling. Mode-locked pulses in the sub $100 \mathrm{fs}$ regime have been reported from these oscillators [9$11]$.

We report on what is to our knowledge the first directly diode pumped cavity dumped laser oscillator emitting femtosecond pulses. Energies beyond $400 \mathrm{~nJ}$ resulting in peak powers well beyond 1 MW have been achieved. The laser is mode-locked in the solitary regime [12, 13] employing dispersive mirrors and a SESAM. Furthermore, from the scientific point of view, this laser system allows a deep insight into femtosecond solitary pulse shaping in the transient regime, and pulsing dynamics such as temporal and spectral breathing of the intracavity pulses have been directly observed.

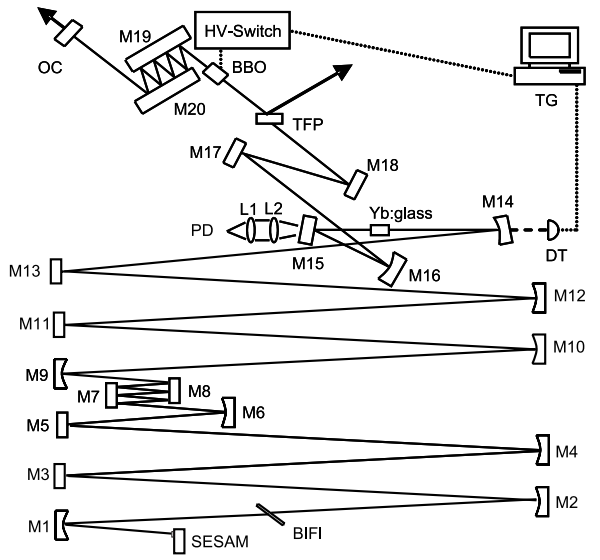

Fig. 1. Schematic of the diode pumped cavity dumped Yb:glass laser oscillator; PD, pump-diode; L1, L2, achromatic lenses; Yb, anti reflection coated Yb:glass laser medium; BIFI birefringent filter; SESAM, semiconductor saturable absorber mirror; OC, $0.3 \%$ output coupling mirror; M15, dichroic mirror; the other mirrors dispersive high reflectors; TFP, thin film polarizer; BBO Pockels cell; HV-Switch, high voltage switch; TG, trigger generator; PD, fast photo diode. 
The laser cavity, shown in Fig.1, was a standard zfold setup. The focus in the active laser medium was generated by two curved mirrors with a focal length of $75 \mathrm{~mm}$ (M14, M16). The focal radius was estimated to be about $40 \mu \mathrm{m}$. The cavity was stretched by use of four curved mirrors with $500 \mathrm{~mm}$ focal length (M2, M4, M10, M12) and two curved mirrors with a focal length of 250mm (M6, M9), which lead to a repetition frequency as low as $21 \mathrm{MHz}$. The gain medium was a $3.5 \mathrm{~mm}$ thick anti-reflection coated Ytterbium doped LG760 phosphate glass pumped by a 976nm laser diode which was focused down to a radius of $65 \mu \mathrm{m}$. At the pump input face of the Yb:LG760 a maximum pump power of $3.2 \mathrm{~W}$ was achieved of which $1.2 \mathrm{~W}$ is transmitted under nonlasing conditions. A SESAM provided for the saturable absorption necessary to mode-lock the laser. Focusing down to a $150 \mu \mathrm{m}$ spot size on the SESAM was done by a curved mirror with $150 \mathrm{~mm}$ focal length. In this setup no damage on the SESAM's surface was observed even when operating the system for hours in dumped operation. In contrast to previous approaches using prisms for dispersion compensation [4-7], specially designed dispersive mirrors were used to generate a sufficient amount of negative dispersion for soliton mode-locking. Each mirror reflection provided a group delay dispersion (GDD) of about $-250 \mathrm{fs}^{2}$ to $-300 \mathrm{fs}^{2}$ and 52 mirror bounces per round trip were employed to get stable solitary operation. The estimated net GDD of the laser cavity was $D=-8100 \mathrm{fs}^{2}$. Because of the high number of reflections needed to generate the GDD, a very high reflectivity of the used mirrors was essential. A birefringent filter [14, 15] was placed in the long laser arm to increase the laser's stability and to allow for wavelength tuning. To avoid excess selfphase modulation (SPM), typically accrued in acousto-optic modulators with a tightly focused fsbeam which is necessary to provide for a dumping duration shorter then the pulse repetition time, we used a BBO electro-optical dumper (Lightgate 03 from Cleveland Crystals Inc.) in the short arm of the cavity. As focusing is not necessary, the electrooptical dumper's SPM does not play an important role. By applying a voltage to the BBO crystal it gets birefringent. Consequently the polarization of the laser beam gets elliptical and a significant amount of the pulse energy is dumped out of the cavity at the thin film polarizer (TFP) [16, 17]. The dumper was controlled by a trigger generator (TG) and a high voltage switch with a maximum output up to $3.6 \mathrm{kV}$ at $206 \mathrm{kHz}$. A voltage of about $2 \mathrm{kV}$ was sufficient to dump more than half of the intra cavity energy. A fast photo diode provided the signal necessary for the synchronization of the trigger generator.
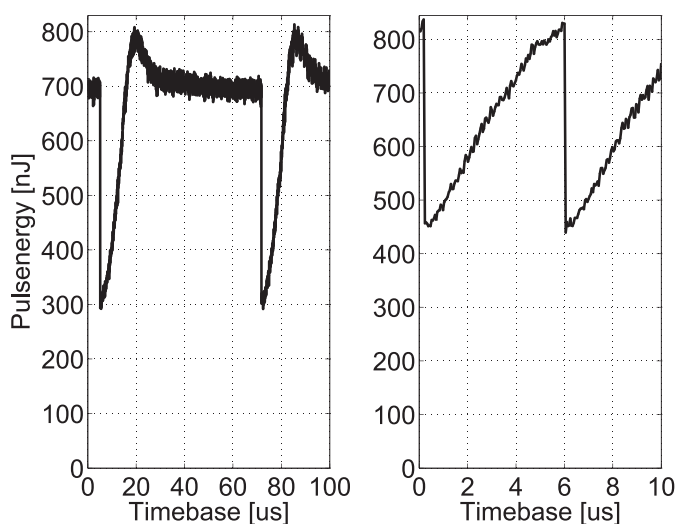

Fig. 2. Intracavity pulse energy between two cavity dumping events. Left and right hand sides show the relaxation at dumping rates of $15 \mathrm{kHz}$ and $173 \mathrm{kHz}$ respectively, note the different time scales.

Behind the TFP, the contrast ratio between the dumped pulses and the weak background was better than 1:500. Fig. 2 shows typical relaxation transients. In the left figure the dumping rate was low enough to let the system reach steady state after each dumping event. The intra cavity dynamics showed a strong damping of the relaxation oscillations in comparison to CW operation of the laser system. This was due to a strong spectral filtering effect while operating the laser at the stability edge, close to $\mathrm{CW}$ breakthrough or close to multiple pulse break-up respectively [18]. In the configuration from the right figure the dumping rate was much higher and the dumping occurs in the transient regime. In both cases it was possible to dump more than 50 percent of the intra cavity energy without losing the stability of operation. The dumped pulse energies per pulse were determined to be more than 400nJ even at repetition frequencies as high as $173 \mathrm{MHz}$.

The full width of the optical spectrum of the dumped pulses were $4 \mathrm{~nm}$, the center wavelength was tunable between $1030 \mathrm{~nm}$ and $1045 \mathrm{~nm}$ without degrading the dumping performance of the system. The duration of the dumped pulses, obtained by background-free autocorrelation was 300 fs, assuming a $\operatorname{sech}^{2}$ pulse shape, resulting in a time bandwidth product of 0.33 , which is close to the Fourier limit. The SPM-parameter $\gamma$ for two passes through both, the $3.5 \mathrm{~mm}$ laser glass and the $36 \mathrm{~mm}$ long BBO pockels cell with spotsizes of $60 \mu \mathrm{m}$ and $400 \mu \mathrm{m}$ radius respectively, was estimated as $\gamma=1.3 \times 10^{-7} / \mathrm{W}$, which leads to the corresponding solitary pulse width of $\tau=3.53|D| /(\gamma w)$ [18], with the intra-cavity pulse energy of $w=700 \mathrm{~nJ}$. The excellent agreement of $\tau=314 \mathrm{fs}$ to the measured 
value proves the clean solitary pulse shaping in the laser system.

Cavity dumping is a periodic disturbance of the system and in certain parameter ranges unstable states exist, where spiking occurs or Q-switched mode-locking is initiated. Due to the long upper state life time of Yb:glass in the millisecond region [10], Q-switch instabilities are a problem to be considered. In the solitary parameter regime, spectral filtering acts contrary to the saturable absorber and thus can suppress Q-switching instabilities and strong relaxation oscillations. Therefore, it was crucial to operate the laser at high power close to CW- or multiple pulse break-up [19]. Due to the periodic disturbance, the pulse shaping dynamics can be directly observed. Between two regions of stable operation namely on one end at low dumping frequency, where the system is allowed to reach steady state, and on the other end at high frequencies, where dumping happens in the transient regime, a region of dynamical instability was identified, where effects such as period doubling sequences in pulse energies have been observed.

In conclusion we have performed what is to our knowledge the first diode pumped cavity-dumped laser oscillator in the femtosecond regime. Pulse durations of $300 \mathrm{fs}$ and pulse energies as high as $400 \mathrm{~nJ}$ have been achieved at relatively high repetition rates of $173 \mathrm{kHz}$. This yields peak powers exceeding 1MW. The laser preserved tunability and short-pulse operation, while increasing the obtained pulse energy at variable repetition frequencies in comparison to a standard oscillator. The prismless design made the system compact. Along with the very cost efficient diode pumping scheme and its high reliability, we believe that a similar system can be a light source for various scientific, industrial and biomedical applications such as material processing, nonlinear spectroscopy etc. In the reported experiments the limitation in pulse energy has been due to the limited gain of the Yb:glass laser material, and the pulse width was limited by the absorber strength. With continued optimization pulse energies in the $\mu \mathrm{J}$ regime can be anticipated in the near future.

The authors would like to thank Franz X. Kärtner and Stefan Mohr for fruitful discussions. This research was funded by the European Union within the contract G1ST-CT-2002-50266 (DACO).

1. G. Cerullo, and S. De Silvestri, "Ultrafast optical parametric amplifiers," Review of Scientific Instruments 74, 1-18 (2003).

2. K. M. Davis, K. Mirura, N. Sugimoto, and K. Hirao, "Writing waveguides in glass with a femtosecond laser," Opt. Lett. 21, 1729-1731 (1996)
3. Osellame, S. Taccheo, M. Marangoni, R. Ramponi, and P. La Porta, D. Polli, S. De Silvestri, and G. Cerullo, "Femtosecond writing of active optical waveguides with astigmatically shaped beams,” JOSA B 20, 1559-1567 (2003)

4. M. Ramaswamy, M. Ulmann, J. Paye and J. G. Fujimoto, "Cavity-dumped femtsecond Kerr-lens mode-locked Ti: $\mathrm{Al}_{2} \mathrm{O}_{3}$ laser,” Opt. Lett. 18, 18221824 (1993)

5. A. Baltuška, Z. Wei, M. S. Pshenichnikov, D. A. Wiersma, Róbert Szipócs, "All-solid-state cavitydumped sub-5-fs laser,” Appl. Phys. B 65, 175188 (1997)

6. M. S. Pshenichnikov, W. P. de Boeij, and D. A. Wiersma, "Generation of 13-fs, 5-MW pulses from a cavity-dumped Ti:sapphire laser,“ Opt. Lett. 19, 572-574, (1994)

7. S. Schneider and A. Stockmann, W. Schüßlbauer, "Self-starting mode-locked cavity-dumped Ti:sapphire laser employing a semiconductor saturable absorber mirror,” Opt. Exp. 6, 220-226 (2000)

8. U. Keller, K. J. Weingarten, F. X. Kärtner, D. Kopf, B. Braun, I. D. Jung, R. Fluck, C. Hönninger, N. Matuschek, and J. Aus der Au, "Semiconductor saturable absorber mirrors (SESAM's) for femtosecond to nanosecond pulse generation in solid-state lasers,” IEEE J. Sel. Top. Quantum Electron. 2, 435 (1996)

9. C. Hönninger, F. Morier-Genoud, M. Moser, and U. Keller, "Efficient and tunable diode-pumped femtosecond Yb:glass lasers," Opt. Lett. 23, 126128 (1998)

10. F. Druon, S. Chénais, P. Raybaut, F. Balembois, and P.Georges, R. Gaumé, G. Aka, and B. Viana, S. Mohr and D. Kopf, "Diode-pumped $\mathrm{Yb}: \mathrm{Sr}_{3} \mathrm{Y}\left(\mathrm{BO}_{3}\right)_{3}$ femtosecond laser,” Opt. Lett. 27, 197-199 (2002)

11. C. Hönninger, R. Paschotta, M. Graf, et al, "Ultrafast ytterbium-doped bulk lasers and laser amplifiers,” Appl. Phys. B 69, 3-17 (1999)

12. F. X. Kärtner, J. Aus der Au, and U. Keller, "Mode-Locking with Slow and Fast Saturable Absorbers - What's the Difference? ," IEEE J. Sel. Top. Quantum Electron. 4, 159-168 (1999)

13. F. X. Kärtner, I. D. Jung, and U. Keller, "Soliton modelocking with saturable absorbers," IEEE J. Sel. Top. Quantum Electron. 2, 540-556, (1996)

14. A. L. Bloom, "Modes of a resonator containing tilted birefringent plates,” J. Opt. Soc. Am. 64, 447-452, (1974)

15. K. Naganuma, G. Lenz, and E. P. Ippen, "Variable Bandwidth Birefringent Filter for Tunable Femtosecond Lasers,” IEEE J. Quantum Electron. 28, (1992) 
16. V. Kubecek, J. Biegert, J. C. Diels and M. R. Kokta, "Practical source of 50ps pulses using a flashlamp pumped Nd:YAG laser and passive allsolid-state pulse control," Opt. Commun. 177, 317-321 (2000)

17. E. Krüger, "High-repetition-rate electro-optic cavity dumping,” Rev. Sci. Instrum., 66, 961-967 (1995)
18. T. Brabec, Ch. Spielmann, and F. Krausz, "Mode locking in solitary lasers,” Opt. Lett. 16, 19611963 (1991)

19. T. R. Schibli, E. R. Thoen, F. X. Kärtner, E. P. Ippen, "Suppression of Q-switched mode locking and break-up into multiple pulses by inverse saturable absorption,” Appl. Phys. B 70, 41-49 (2000) 